\title{
Idiopathic Nephrotic Syndrome
}

National Cancer Institute

\section{Source}

National Cancer Institute. Idiopathic Nephrotic Syndrome. NCI Thesaurus. Code

C122796.

Nephrotic syndrome for which no cause has been identified. 